\title{
Surgery and Chemotherapy versus Chemotherapy Only in Older Persons with Primary Intestinal Diffuse Large B-Cell Lymphoma
}

\author{
Limei Zhang, 1,2,* He Huang, 1,3,* \\ Zhao Wang, (D) ${ }^{1,3, *}$ Xiaojie Fang, ${ }^{1,3}$ \\ Huangming Hong, 4 \\ Yungchang Chen, ${ }^{4}$ Fangfang $\mathrm{Li}^{1,3}$ \\ Yuyi Yao,, 3 Zegeng Chen,, \\ Fei Pan, ${ }^{\text {I,3 Xiaogian Li, }}$ \\ Meiting Chen,, 3 \\ Robert Peter Gale, ${ }^{5}$ Yang Liang, , 2,* \\ Tongyu Lin 1,3,4,* \\ 'Sun Yat-sen University Cancer Center, State \\ Key Laboratory of Oncology in South China, \\ Collaborative Innovation Center for Cancer \\ Medicine, Guangzhou, People's Republic of \\ China; ${ }^{2}$ Department of Hematologic \\ Oncology, Sun Yat-sen University Cancer \\ Center, State Key Laboratory of Oncology in \\ South China, Guangzhou, People's Republic of \\ China; ${ }^{3}$ Department of Medical Oncology, Sun \\ Yat-sen University Cancer Center, State Key \\ Laboratory of Oncology in South China, \\ Guangzhou, People's Republic of China; \\ ${ }^{4}$ Department of Medical Oncology, Sichuan \\ Cancer Hospital and Institute, Sichuan Cancer \\ Center, School of Medicine, University of \\ Electronic Science and Technology of China, \\ Chengdu, People's Republic of China; \\ ${ }^{5}$ Department of Immunology and \\ Inflammation, Haematology Research Centre, \\ Imperial College London, London, UK
}

*These authors contributed equally to this work

Correspondence: Tongyu Lin Department of Medical Oncology, Sun Yat-sen University Cancer Center, State Key Laboratory of Oncology in South China, 65I Dongfeng Road East,

Guangzhou, Guangdong, 510060, People's Republic of China

Email linty@sysucc.org.cn

Yang Liang

Department of Hematologic Oncology, Sun Yat-sen University Cancer, State Key Laboratory of Oncology in South China, 65I Dongfeng Road East, Guangzhou, Guangdong, 510060, People's Republic of China

Email liangyang@sysucc.org.cn
Background: The management of primary intestinal diffuse large B cell lymphoma (PIDLBCL) in elderly patients (aged $>60$ years) remains controversial. We conducted a retrospective study to assess the efficacy of different treatment strategies and prognostic factors for elderly Chinese patients with PI-DLBCL.

Patients and Methods: Forty-six untreated elderly patients with PI-DLBCL were included in this retrospective study. Twenty-four patients were treated with surgery (prior to chemotherapy) plus chemotherapy (SCT). The other 22 patients did not undergo surgery before chemotherapy (CT).

Results: Patients treated with SCT had a higher overall response rate of $91.7 \%$ than patients receiving $\mathrm{CT}$, but the difference between groups was not significant $(P=0.581)$. Regarding survival, SCT resulted in a greater 3-year overall survival (OS) rate $(87.3 \%$ vs $56.9 \%$, $P=0.130)$ and significantly higher 3 -year event-free survival (EFS) rate $(74.1 \%$ vs $27.3 \%$, $P=0.002)$ than $\mathrm{CT}$. The univariate analysis showed that male sex, advanced Lugano stage, poor performance status and chemotherapy alone were associated with a shorter EFS. Only the male sex was correlated with a shorter OS. The multivariate analysis showed that sex $(P=0.040)$ and treatment strategy $(P=0.022)$ were independent prognostic factors for EFS.

Conclusion: Surgery plus chemotherapy produced a better outcome for EFS, but not OS, than chemotherapy alone in elderly Chinese patients with PI-DLBCL.

Keywords: PI-DLBCL, surgery, chemotherapy, survival, prognostic factors

\section{Introduction}

The gastrointestinal (GI) tract is the most common site of extranodal non-Hodgkin's lymphoma (NHL). ${ }^{1}$ Compared to the stomach, the intestine is the second most common site of primary GI lymphomas. ${ }^{2}$ Primary NHL of the GI tract accounts for $10-15 \%$ of all NHL cases and $1-4 \%$ of all GI tumours. ${ }^{3,4}$ The most common histological types of NHL in the GI tract are diffuse large B-cell lymphoma (DLBCL) and mucosa-associated lymphoid tissue lymphoma (MALT). ${ }^{5,6}$ DLBCL is the most common subtype of primary intestinal lymphoma. ${ }^{7}$

According to some previous studies, the combination of rituximab, cyclophosphamide, doxorubicin, vincristine and prednisone (R-CHOP) is the standard treatment for nodal DLBCL, ${ }^{8-11}$ but the best treatment for PI-DLBCL remains controversial because of the lack of prospective randomized studies with a large sample size. The therapeutic methods for PI-DLBCL include surgery, chemotherapy and targeted therapy or a combination of these treatments. Compared to 
a single modality, some studies have shown that surgery plus immunochemotherapy provides a better survival benefit for patients with PI-DLBCL. ${ }^{12,13}$ Because a standard treatment for PI-DLBCL is unavailable, the management is even less clear for elderly patients (aged $>60$ years). The organ functions and physical performance of elderly patients exhibit a decreasing trend over time. In addition, these patients have a lower tolerance for surgery and chemotherapy and more basic diseases and comorbidities than younger patients. Many uncertain factors are likely to occur during treatment. Considering these factors, individualized assessments and treatments are necessary for elderly patients with PIDLBCL.

Therefore, we conducted a retrospective study focusing on the clinical characteristics, efficacy of different treatment strategies and prognostic factors of 46 elderly patients with PI-DLBCL aged 60 years or older who were treated at our institute from February 2007 to November 2019.

\section{Patients and Methods}

Patients aged more than 60 years who were newly diagnosed with primary intestinal DLBCL between February 2007 and November 2019 at Sun Yat-sen University Cancer Center were enrolled in this retrospective study. DLBCL was defined according to the World Health Organization (WHO) classification of Tumours of Haematopoietic and Lymphoid Tissue (2008). To date, the best method for distinguishing primary intestinal DLBCL from systemic DLBCL involving the intestine remains unclear. In our study, patients who presented with predominant intestinal lesions were regarded as having primary intestinal DLBCL. ${ }^{1,14,15}$ Computed tomography (CT) or positron emission tomography (PET) scans should be performed before treatment. The stage of the disease was based on the Lugano staging system for gastrointestinal (GI) lymphomas. ${ }^{16}$ The definition of bulky disease was a mass diameter $\geq 10 \mathrm{~cm}$.

Clinicians decided which treatment each patient received. Patients were divided into two groups based on whether they received surgery as the initial treatment for DLBCL, followed by a first-line regimen with cyclophosphamide, vincristine, doxorubicin, prednisone and rituximab (R-CHOP). All patients received a standard dose of R-CHOP every 21 days. After treatment, all patients underwent a therapeutic evaluation using PET-CT. The revised response criteria for malignant lymphoma were used to evaluate the response of patients with PI-DLBCL to treatment. ${ }^{17}$

\section{Statistical Analysis}

Statistical analyses were performed with the SPSS version 22 and GraphPad Prism 7 software packages. The $\chi^{2}$ test was used to compare the clinical features and response rates between the two groups. Kaplan-Meier curves were constructed to estimate event-free survival (EFS) and overall survival (OS). The survival curves were compared between two groups using the Log rank test. Univariate and multivariate analyses were performed to identify prognostic factors for survival,which were expressed as hazard ratio (HR) with $95 \%$ confidence interval (CI). All tests were two-sided, and a $P$ value less than 0.05 was considered statistically significant. EFS was calculated from the date of diagnosis to the date of progression, relapse, severe complications (such as intestinal perforation) and death from any cause or the date of the last follow-up. OS was calculated from the date of diagnosis to the date of death from any cause or the last follow-up.

\section{Results}

\section{Patient Characteristics}

The characteristics of the 46 enrolled patients are shown in Table 1. The median follow-up duration was 35 months (range 3-113 months). The median age of the patients was 67 years (range, 61-81 years), and the male-to-female ratio was 2.07:1 (31:15). Most patients had a good performance status (Eastern Cooperative Oncology Group,ECOG 0/1, 95.7\%). A greater number of patients were diagnosed with advanced disease (II-2/II-E/IV) than with early-stage disease (I/II-1). The distribution of the lesions in the intestine was as follows: small intestine $(\mathrm{n}=13,28.3 \%)$, ileocecum $(\mathrm{n}=8$, $17.4 \%)$, large intestine ( $\mathrm{n}=14,30.4 \%)$, and multiple regions $(\mathrm{n}=11,23.9 \%)$. Twenty-four patients underwent surgery before receiving R-CHOP chemotherapy (SCT), while 22 patients were treated with R-CHOP chemotherapy without surgery (CT) as the initial treatment. Regarding the relative dose intensity of each group, both the SCT group and CT group received a median number of $6 \mathrm{R}-\mathrm{CHOP}$ chemotherapy cycles (range, 2-8). Patients in both the SCT and CT groups received the R-CHOP regimen as the first-line chemotherapy regimen. None of the patients received radiotherapy or radiotherapy alone, and every patient received at least two cycles of R-CHOP. A comparison of the clinical features between the two groups is listed in Table 1. The number of patients with increased serum $\beta 2$-microglobulin levels was significantly lower in the SCT group than in the CT group $(P=0.033$, Table 1$)$. Because resection of the 
Table I Clinical Characteristics of Patients $(n=46)$

\begin{tabular}{|c|c|c|c|c|}
\hline Characteristic & Total $(n=46)$ & $\operatorname{SCT}(n=24)$ & $C T(n=22)$ & $P$ value \\
\hline Median age(range) & $67(6 I-8 I)$ & $65.5(6 I-80)$ & $67(6 I-8 I)$ & 0.432 \\
\hline \multicolumn{5}{|l|}{ Sex } \\
\hline Male & 31 & 15 & 16 & 0.460 \\
\hline Female & 15 & 9 & 6 & \\
\hline \multicolumn{5}{|l|}{ Performance status } \\
\hline ECOG $0 / 1$ & 44 & 24 & 20 & 0.432 \\
\hline $\mathrm{ECOG} \geq 2$ & 2 & 0 & 2 & \\
\hline \multicolumn{5}{|l|}{ Serum LDH level } \\
\hline Normal & 30 & 16 & 14 & 0.673 \\
\hline Increased & 15 & 7 & 8 & \\
\hline Missing & 1 & 1 & & \\
\hline \multicolumn{5}{|l|}{ Serum $\beta 2-M G$} \\
\hline Normal & 18 & 12 & 6 & 0.033 \\
\hline Increased & 19 & 6 & 13 & \\
\hline Missing & 9 & 6 & 3 & \\
\hline \multicolumn{5}{|l|}{ Albumin } \\
\hline Decreased & 32 & 17 & 15 & 0.845 \\
\hline Normal & 14 & 7 & 7 & \\
\hline \multicolumn{5}{|l|}{ B symptoms } \\
\hline Absent & 35 & 18 & 17 & 0.905 \\
\hline Present & 10 & 6 & 4 & \\
\hline Missing & I & & 1 & \\
\hline \multicolumn{5}{|l|}{ IPI } \\
\hline $0-1$ & 24 & 15 & 9 & 0.102 \\
\hline$\geq 2$ & 21 & 8 & 13 & \\
\hline Missing & 1 & I & & \\
\hline \multicolumn{5}{|l|}{ Lugano stage } \\
\hline$|/| I-I$ & 16 & 11 & 5 & 0.100 \\
\hline II-2/II-E/IV & 30 & 13 & 17 & \\
\hline \multicolumn{5}{|l|}{ Location } \\
\hline Small intestinal & 13 & 7 & 6 & 0.698 \\
\hline lleocecus & 8 & 5 & 3 & \\
\hline Large intestinal & 14 & 8 & 6 & \\
\hline Multiregion & II & 4 & 7 & \\
\hline \multicolumn{5}{|l|}{ Bulky disease } \\
\hline Absent & 33 & 20 & 13 & 0.068 \\
\hline Present & 13 & 4 & 9 & \\
\hline \multicolumn{5}{|l|}{ Hans's subtype } \\
\hline GCB & 18 & 12 & 6 & 0.228 \\
\hline N-GCB & 23 & 11 & 12 & \\
\hline Missing & 5 & 1 & 4 & \\
\hline \multicolumn{5}{|l|}{ Intestinal perforation } \\
\hline Absent & 41 & 24 & 17 & 0.046 \\
\hline Present & 5 & 0 & 5 & \\
\hline
\end{tabular}

(Continued) 
Table I (Continued).

\begin{tabular}{|c|c|c|c|c|}
\hline Characteristic & Total $(n=46)$ & $\operatorname{SCT}(n=24)$ & $C T(n=22)$ & $P$ value \\
\hline \multicolumn{5}{|c|}{ Extranodal involvement } \\
\hline$<2$ & 36 & 20 & 16 & 0.608 \\
\hline$\geq 2$ & 10 & 4 & 6 & \\
\hline \multicolumn{5}{|l|}{ R-CHOP cycles } \\
\hline$<6$ & 20 & 11 & 9 & 0.736 \\
\hline$\geq 6$ & 26 & 13 & 13 & \\
\hline \multicolumn{5}{|c|}{ Reason for surgery } \\
\hline Mass resection & & 12 & & \\
\hline Diagnostic & & 11 & & \\
\hline Perforation & & 1 & & \\
\hline \multicolumn{5}{|l|}{ Radiotherapy } \\
\hline Done & 0 & 0 & 0 & 1.000 \\
\hline Not done & 46 & 24 & 22 & \\
\hline \multicolumn{5}{|c|}{$\begin{array}{l}\text { Complications after } \\
\text { surgery }\end{array}$} \\
\hline Present & I & I & & \\
\hline Absent & 45 & 23 & & \\
\hline \multicolumn{5}{|l|}{ Comorbidity } \\
\hline Absent & 21 & 11 & 10 & 0.979 \\
\hline Present & 25 & 13 & 12 & \\
\hline
\end{tabular}

Abbreviations: ECOG, Eastern Cooperative Oncology Group; LDH, lactate dehydrogenase; MG, microglobulin; IPI, international prognostic index; SCT, Surgery +Chemotherapy; CT, Chemotherapy; GCB, germinal center B-cell; R-CHOP, Rituximab in combination with cyclophosphamide, adriamycin, vincristine, and prednisolone.

intestinal lesions was performed, the SCT group had a lower rate of intestinal perforation $(0 / 24)$ than the CT group $(22.8 \%, 5 / 22)$. Most patients recovered well after surgery, and $54.3 \%$ of the patients $(25 / 46)$ presented comorbidities. Detailed information on comorbidities between the two groups is listed in Table 2. Most patients in the two groups had hypertension, diabetes and chronic hepatitis B. Other clinical features, such as sex, performance status, serum LDH level, albumin level, B symptoms, IPI score, Lugano stage, location of the disease, bulky disease, Hans's subtype and extranodal involvement, did not differ significantly between the two groups. Five patients who experienced

Table 2 The Detailed Comparison Between Two Groups in Main Comorbidity

\begin{tabular}{|l|c|c|}
\hline Comorbidity & SCT( $\mathbf{n}=\mathbf{2 4})$ & $\mathbf{C T}(\mathbf{n}=\mathbf{2 2})$ \\
\hline Hypertension disease & 5 & 3 \\
Diabetes & 3 & 4 \\
Chronic hepatitis B & 4 & 2 \\
Lumbar disc herniation & $\mathrm{I}$ & 2 \\
Chronic cholecystitis & $\mathrm{I}$ & $\mathrm{I}$ \\
\hline
\end{tabular}

intestinal perforation during chemotherapy underwent emergency surgery. The characteristics of patients who developed intestinal perforation during chemotherapy are shown in Table 3.

\section{Treatment Outcomes and Prognostic Factors}

The overall response rate of the SCT group was $91.7 \%$ $(22 / 24)$, which was higher than that of the CT group $(81.8 \%, 18 / 22)$, after receiving the first-line regimen, but this difference was not significant $(P=0.581)$. The complete remission (CR) rate was higher in the SCT group

Table 3 Characteristics of Patients Developed Intestinal Perforation During Chemotherapy

\begin{tabular}{|l|l|l|l|l|l|}
\hline No & Age & Sex & Stage & Surgery & Status \\
\hline I & 8 I & Male & IV & Partial ileectomy & Alive \\
2 & 73 & Male & IV & Partial ileectomy & Dead \\
3 & 70 & Female & IV & Right hemicolectomy & Dead \\
4 & 65 & Male & IV & Partial ileectomy & Alive \\
5 & 65 & Male & IV & Partial ileectomy & Alive \\
\hline
\end{tabular}


Table 4 Response of Patients Based on Treatment Strategy $(n=46)$

\begin{tabular}{|l|l|l|l|l|l|l|l|}
\hline \multicolumn{2}{|l|}{ Relapse and Response, $\mathbf{n}(\%)$} & P value \\
\hline Group & Total & Relapse & CR & PR & SD/PD & ORR \\
\hline SCT & 24 & $4(16.7)$ & $19(79.2)$ & $3(12.5)$ & $2(8.3)$ & $22(91.7)$ & $0.581($ ORR) \\
CT & 22 & $7(31.8)$ & $15(68.2)$ & $3(13.6)$ & $4(18.2)$ & $18(81.8)$ & \\
\hline
\end{tabular}

Abbreviations: CR, complete response; PR, partial response; SD, stable disease; PD, progressive disease; ORR, overall response rate.

(79.2\%) than in the CT group (68.2\%), but this difference was also not statistically significant $(P=0.397)$. Regarding the relapse rate, the SCT group had a significantly lower relapse rate $(16.7 \%)$ than the $\mathrm{CT}$ group (31.8\%) $(P=0.229)$. Patient responses based on the treatment strategy are shown in Table 4. Regarding survival, the 3-year OS rate was $87.3 \%$ in the SCT group and $56.9 \%$ in the CT group ( $P=0.130$, Figure 1A). The 3-year EFS rate of the SCT group was $74.1 \%$, which was significantly higher than that of the CT group $(27.3 \%, P=0.002$, Figure $1 \mathrm{~B})$.

The univariate analysis showed that sex was the only prognostic factor for OS $(P=0.045)$. A multivariate analysis of OS was not performed, as only sex was significant in the univariate analysis. For EFS, the univariate analysis indicated that sex (HR: 4.635 [95\% CI: 1.369-15.694]; $P=0.014$ ), performance status (HR: 0.225 [95\% CI: $0.051-$ 0.993]; $P=0.049$ ), Lugano stage (HR: 0.366 [95\% CI: $0.135-$ 0.992]; $P=0.048$ ), IPI (HR: 0.313 [95\% CI: 0.132-0.744]; $P=0.009$ ) and treatment strategy (HR: 0.269 [95\% CI: 0.109 $0.664] ; P=0.004)$ were prognostic factors for EFS. However, the multivariate analysis indicated that only sex (HR: 3.834 [95\% CI: $1.064-13.815] ; P=0.040)$ and treatment strategy (HR: 0.333 [95\% CI: 0.129-0.855]; $P=0.022$ ) were independent prognostic factors for EFS.

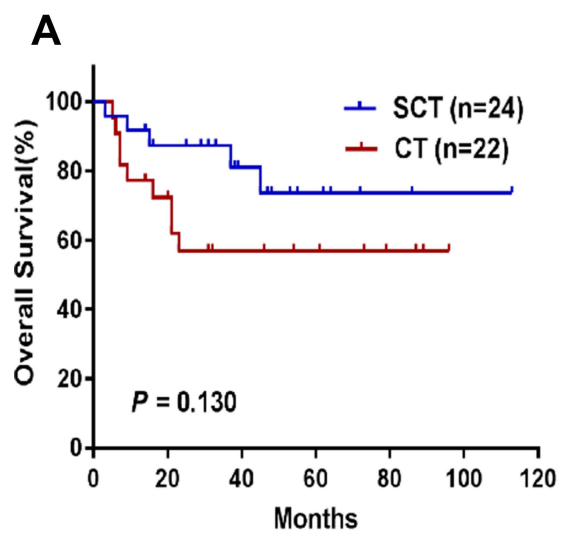

Univariate and multivariate analyses of the prognostic factors for EFS are shown in Figure 2. Among the 46 patients, the 3-year EFS rate of females was $79.4 \%$, which was significantly higher than that of males (37.6\%, HR: 4.528 [95\% CI: 1.969-10.410]; $P=0.006$ ). Patients with early-stage disease (I/II-1) had a better 3 -year EFS rate $(74.5 \%)$ than those with advanced disease (II-2/II-E/IV) (38.9\%, HR: 0.372 [95\% CI: 0.163-0.850]; $P=0.037)$. Patients with a low IPI score $(0-1)$ also had a higher 3-year EFS rate than those with a high IPI score ( $\geq 2$ ) (70.3\% vs 27.2\%, HR: 0.319 (95\% CI: 0.137-0.743); $P=0.005)$. EFS rates of patients stratified according to the different risk factors are shown in Figure 3.

\section{Discussion}

In our study, the SCT group achieved a significantly higher 3 -year EFS rate than the CT group (74.1\% vs $27.3 \%$, $P=0.002$ ), but the 3 -year OS rate did not show a similar trend $(87.3 \%$ vs $56.9 \%, P=0.130)$. The OS of the two groups was similar to that observed in a previous study. ${ }^{18}$ In addition, the $\mathrm{CR}$ rate and overall response rate did not differ significantly between the SCT group and $\mathrm{CT}$ group. The SCT group had a higher $\mathrm{CR}$ rate $(79.2 \%)$ and a lower relapse rate (16.7\%) than the CT

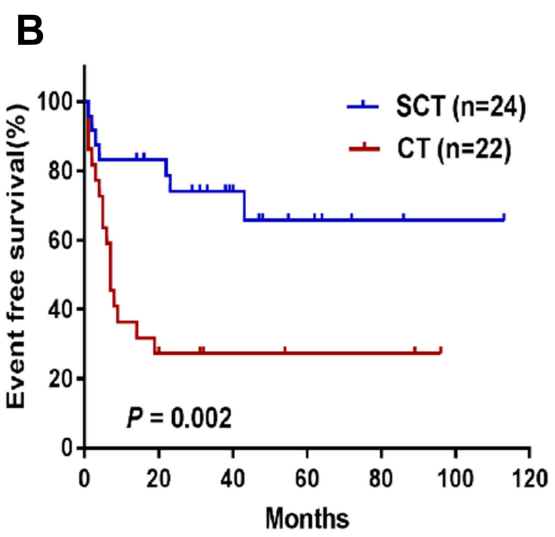

Figure I Survival of patients according to different treatment strategy. (A) OS; (B) EFS. Abbreviations: SCT, surgery+chemotherapy; CT, chemotherapy. 


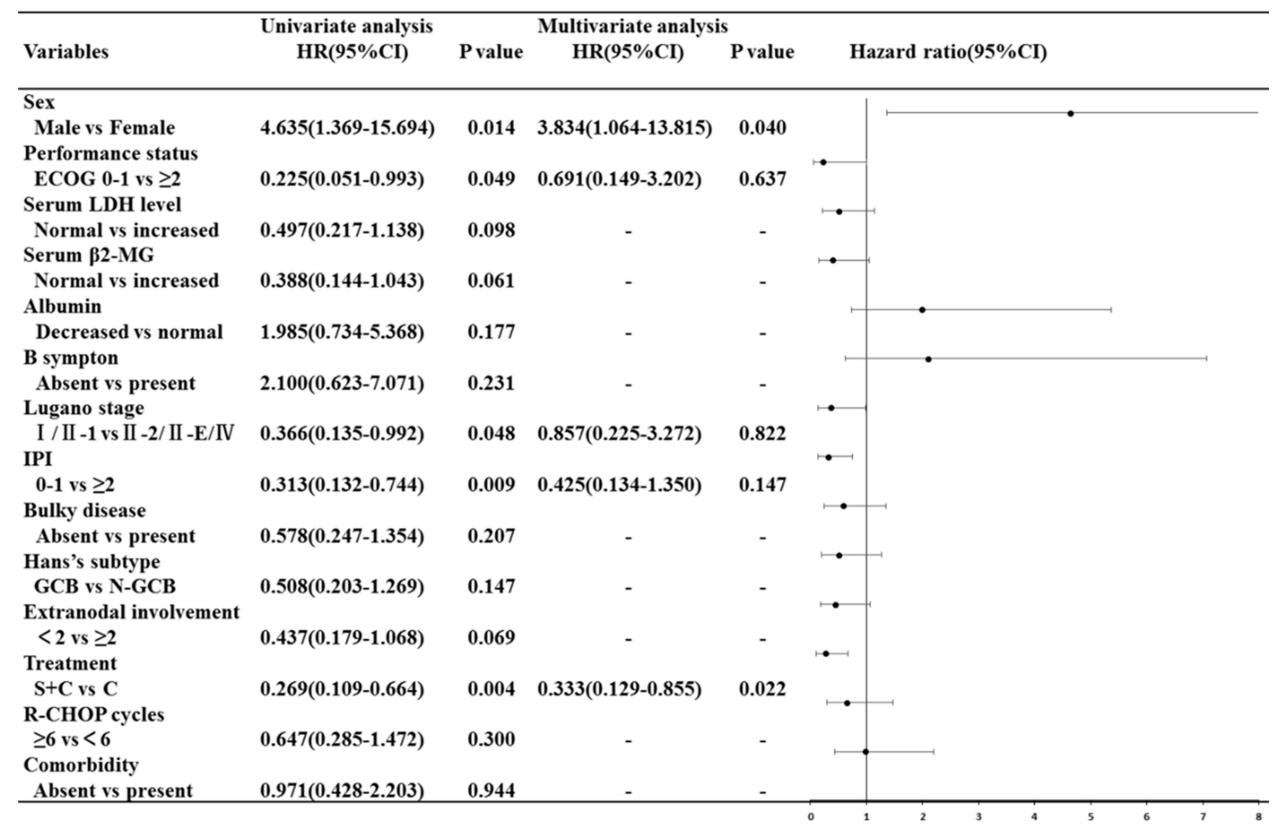

Figure 2 Results of univariate and multivariate Cox regression.

A

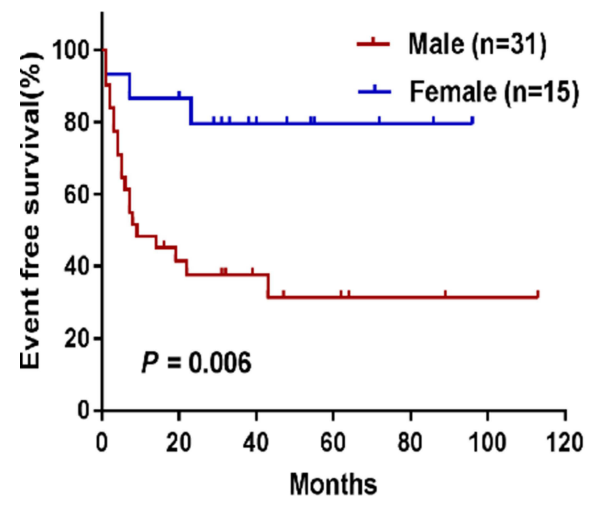

C

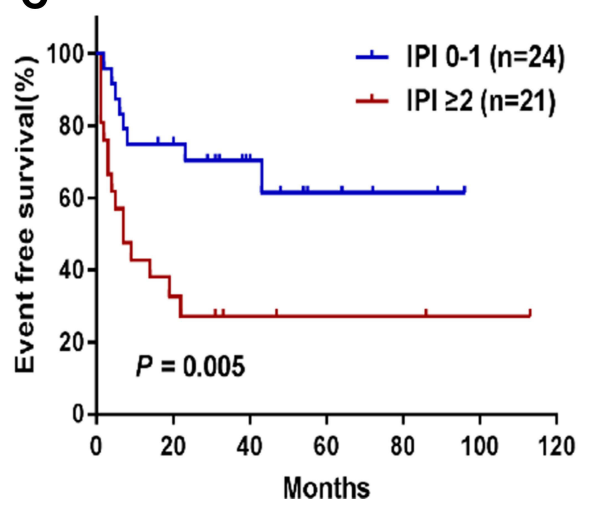

B

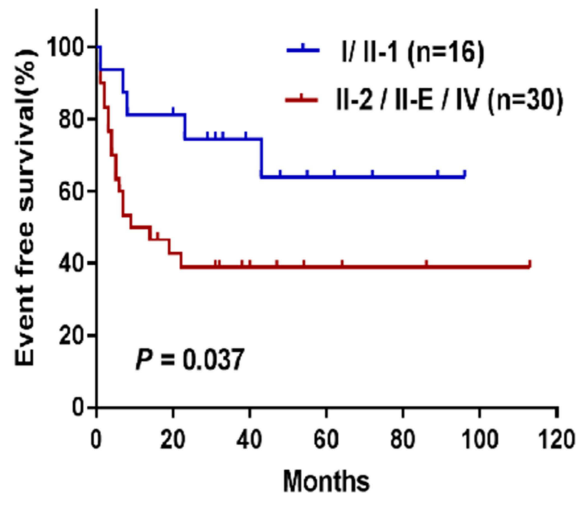


group, which had a $\mathrm{CR}$ rate of $68.2 \%$ and relapse rate of $31.8 \%$, but this difference was not significantly different. This finding may be due to the small sample size of the study. The CR and relapse rates reported here are also similar to those reported in a Korean study. ${ }^{13}$ Due to the resection of intestinal lesions, the chance of intestinal perforation may decrease the possibility of tumour metastases to some extent. In addition, because of the difficulty in discriminating residual lesions in cases of bowel wall thickening, underestimation of the remaining lesions might be another explanation for the higher relapse rate in the CT group. ${ }^{13}$ When bulky disease exists in the abdomen, chemotherapeutic drugs are unable to easily reach the inside of the tumour. Therefore, the SCT group exhibited a better EFS rate and lower relapse rate than the CT group.

In the past 10 years, nonsurgical treatment has shown better efficacy and lower toxicity than surgical treatment, and the treatment mode for primary gastric lymphoma has shifted from surgical treatment to nonsurgical treatment.,19-21 Currently, surgery is usually used in cases of gastric perforation, bleeding, or obstruction due to tumours or for the resection of refractory lesions in patients with gastric lymphoma. However, unlike gastric DLBCL, surgery is usually performed because of perforation or obstruction of the intestine before diagnosis. In addition, as the use of colonoscopy for biopsy may be difficult to determine a clear pathologic diagnosis, surgery is often necessary, even in the absence of an emergent condition or other complications related to intestinal lesions. Compared to patients with a thick gastric wall, those with a thin intestinal wall are more likely to develop perforation. Therefore, in cases of intestinal perforation, preventative resection of intestinal lesions before chemotherapy is usually performed. Based on the reasons mentioned above, surgery is more commonly performed to treat PI-DLBCL. Because of the floating features of the intestine, radiation therapy is rarely used to treat PI-DLBCL.

Although surgery is commonly performed for PIDLBCL, surgical resection of the primary mass is still controversial because DLBCL is curable using chemotherapy. Surgery may increase the incidence of related complications and reduce the quality of life of patients, especially elderly patients. Because of the specific characteristics of elderly patients, such as a weakened condition, organ dysfunction, underlying diseases, and a poor performance status, the treatment of lymphoma with the issues noted above experienced by elderly patients must be balanced. Thus, the treatment of elderly patients with lymphoma is not as simple as that of younger patients. Sometimes elderly patients refuse subsequent chemotherapy because of the physical and psychological pain related to surgery. Thus, the following cycle of chemotherapy will be interrupted or delayed as a result of comorbidities or complications. According to a retrospective Korean study of 345 patients with PIDLBCL, surgery-associated deterioration of quality of life (QOL) may be acceptable, and the survival benefits and lower relapse rate of surgery plus chemotherapy may outweigh the negative effects of surgery on QOL. ${ }^{13}$

In our study, the ratio of males to females was $2: 1$ (31:15), which was similar to that of previous studies. ${ }^{1,12}$ Thus, PIDLBCL has a male preponderance. The univariate analysis showed that sex was the only prognostic factor for OS, and thus we did not perform a multivariate analysis. Other factors, such as stage, performance and sex, were not prognostic factors. However, a large-scale retrospective study based on the SEER database showed that age, Ann Arbor stage and marital status were independent prognostic factors for OS. ${ }^{22}$ The possible explanation for this discrepancy is that our sample size was small compared to that of the previous study. However, unlike the previous study, our study focused on elderly patients with PI-DLBCL and did not include patients with primary gastric DLBCL, resulting in the enrolment of fewer patients in the present study. The multivariate analysis revealed that sex, performance status, stage and IPI score were independent prognostic factors for EFS.

\section{Conclusions}

In conclusion, patients who underwent surgery prior to chemotherapy achieved a significantly better EFS. The SCT group also showed a better response rate, lower relapse rate and higher OS rate, but these differences were not statistically significant. Our study was a retrospective study with a small sample size because of the low incidence of PIDLBCL and our focus on the elderly population. Large-scale prospective clinical studies are needed in the future to further explore the best mode of surgery in combination with chemotherapy for elderly patients with PI-DLBCL.

\section{Ethics Statement}

The study was approved by the Institutional Review Board and Ethics Committee of Sun Yat-sen University Cancer Center. Our study was a retrospective study, and thus the ethics committee exempted the informed consent requirement for patients. Our study adheres to the principles of the Declaration of Helsinki. All included patient data were confidential. 


\section{Author Contributions}

TYL and YL designed the study. LMZ, HH and ZW collected and analysed the data. LMZ, HH and RPG wrote and revised the manuscript. All authors contributed to data analysis, drafting or revising the article, have agreed on the journal to which the article will be submitted, gave final approval of the version to be published, and agree to be accountable for all aspects of the work.

\section{Disclosure}

Yang Liang is supported in part by Sun Yat-sen University Start-up Funding, Grant 201603, the Program for Guangdong Introducing Innovative and Entrepreneurial Teams (2017ZT07S096) and the National Natural Science Foundation of China (Grant No. 81873428). RPG acknowledges support from the National Institute of Health Research (NIHR) Biomedical Research Centre funding scheme. RPG also reports personal fees from Ascentage Pharma Group, BeiGene LTD, Kite Pharma, Inc, Fusion Pharma LLC, La Jolla NanoMedical, Mingsight Pharmaceuticals, Prolacta Bioscience, CStone Pharmaceuticals, FFF Enterprises, Inc., and Antegene Biotech, LLC, outside the submitted work. The authors report no other conflicts of interest in this work.

\section{References}

1. d'Amore F, Brincker H, Grønbaek K, et al. Non-Hodgkin's lymphoma of the gastrointestinal tract: a population-based analysis of incidence, geographic distribution, clinicopathologic presentation features, and prognosis. Danish Lymphoma Study Group. J Clin Oncol. 1994;12 (8):1673-1684. doi:10.1200/jco.1994.12.8.1673

2. Zucca E, Roggero E, Bertoni F, Cavalli F. Primary extranodal nonHodgkin's lymphomas. Part 1: gastrointestinal, cutaneous and genitourinary lymphomas. Ann Oncol. 1997;8(8):727-737. doi:10.1023/ a: 1008282818705

3. Psyrri A, Papageorgiou S, Economopoulos T. Primary extranodal lymphomas of stomach: clinical presentation, diagnostic pitfalls and management. Ann Oncol. 2008;19(12):1992-1999. doi:10.1093/ annonc/mdn 525

4. Loehr WJ, Mujahed Z, Zahn FD, et al. Primary lymphoma of the gastrointestinal tract: a review of 100 cases. Ann Surg. 1969;170 (2):232-238. doi:10.1097/00000658-196908000-00011

5. Koch P, Del Valle F, Berdel WE, et al. Primary gastrointestinal nonHodgkin's lymphoma: I. Anatomic and histologic distribution, clinical features, and survival data of 371 patients registered in the German Multicenter Study GIT NHL 01/92. J Clin Oncol. 2001;19 (18):3861-3873. doi:10.1200/jco.2001.19.18.3861

6. Papaxoinis G, Papageorgiou S, Rontogianni D, et al. Primary gastrointestinal non-Hodgkin's lymphoma: a clinicopathologic study of 128 cases in Greece. A Hellenic Cooperative Oncology Group study (HeCOG). Leuk Lymphoma. 2006;47(10):2140-2146. doi:10.1080/ 10428190600709226

7. Nakamura S, Matsumoto T, Iida M, et al. Primary gastrointestinal lymphoma in Japan: a clinicopathologic analysis of 455 patients with special reference to its time trends. Cancer. 2003;97(10):2462-2473. doi: $10.1002 /$ cncr. 11415
8. Feugier P, Van Hoof A, Sebban C, et al. Long-term results of the R-CHOP study in the treatment of elderly patients with diffuse large B-cell lymphoma: a study by the Groupe d'Etude des Lymphomes de l'Adulte. JClin Oncol. 2005;23(18):4117-4126. doi:10.1200/JCO.2005.09.131

9. Pfreundschuh M, Kuhnt E, Trümper L, et al. CHOP-like chemotherapy with or without rituximab in young patients with good-prognosis diffuse large-B-cell lymphoma: 6-year results of an open-label randomised study of the MabThera International Trial (MInT) Group. Lancet Oncol. 2011;12(11):1013-1022. doi:10.1016/s1470-2045(11) 70235-2

10. Coiffier B, Lepage E, Briere J, et al. CHOP chemotherapy plus rituximab compared with $\mathrm{CHOP}$ alone in elderly patients with diffuse large-B-cell lymphoma. $N$ Engl $J$ Med. 2002;346(4):235-242. doi:10.1056/NEJMoa011795

11. Kako S, Oshima K, Sato M, et al. Clinical outcome in patients with small-intestinal non-Hodgkin lymphoma. Leuk Lymphoma. 2009;50 (10):1618-1624. doi:10.1080/10428190903147629

12. Ibrahim EM, Ezzat AA, El-Weshi AN, et al. Primary intestinal diffuse large B-cell non-Hodgkin's lymphoma: clinical features, management, and prognosis of 66 patients. Ann Oncol. 2001;12(1):53-58. doi:10.1023/a:1008389001990

13. Kim SJ, Kang HJ, Kim JS, et al. Comparison of treatment strategies for patients with intestinal diffuse large B-cell lymphoma: surgical resection followed by chemotherapy versus chemotherapy alone. Blood. 2011;117(6):1958-1965. doi:10.1182/blood-2010-06-288480

14. Lewin KJ, Ranchod M, Dorfman RF. Lymphomas of the gastrointestinal tract: a study of 117 cases presenting with gastrointestinal disease. Cancer. 1978;42(2):693-707. doi:10.1002/1097-0142(197808)42:2<693::AID-CNCR2820420241>3.0.CO;2-J

15. Herrmann R, Panahon AM, Barcos MP, et al. Gastrointestinal involvement in non-Hodgkin's lymphoma. Cancer. 1980;46(1):215-222. doi:10.1002/1097-0142(19800701)46:1<215::AID-

CNCR2820460136>3.0.CO;2-6

16. Rohatiner A, d'Amore F, Coiffier B, et al. Report on a workshop convened to discuss the pathological and staging classifications of gastrointestinal tract lymphoma. Ann Oncol. 1994;5(5):397-400. doi:10.1093/oxfordjournals.annonc.a058869

17. Cheson BD, Pfistner B, Juweid ME, et al. Revised response criteria for malignant lymphoma. J Clin Oncol. 2007;25(5):579-586. doi:10.1200/jco.2006.09.2403

18. Zhao F, Qin Y, Yang J, et al. R-CHOP immunochemotherapy plus surgery is associated with a superior prognosis in Chinese primary intestinal diffuse large B-cell lymphoma. Asia Pac J Clin Oncol. 2020;16(6):385-391. doi:10.1111/ajco.13396

19. Avilés A, Nambo MJ, Neri N, et al. The role of surgery in primary gastric lymphoma: results of a controlled clinical trial. Ann Surg. 2004;240(1):44-50. doi:10.1097/01.sla.0000129354.31318.f1

20. Koch P, del Valle F, Berdel WE, et al. Primary gastrointestinal nonHodgkin's lymphoma: II. Combined surgical and conservative or conservative management only in localized gastric lymphoma-results of the prospective German Multicenter Study GIT NHL 01/92. J Clin Oncol. 2001;19(18):3874-3883. doi:10.1200/jco.2001.19.18.3874

21. Binn M, Ruskoné-Fourmestraux A, Lepage E, et al. Surgical resection plus chemotherapy versus chemotherapy alone: comparison of two strategies to treat diffuse large B-cell gastric lymphoma. Ann Oncol. 2003;14(12):1751-1757. doi:10.1093/annonc/mdg495

22. Wang J, Zhou M, Zhou R, et al. Nomogram for predicting the overall survival of adult patients with primary gastrointestinal diffuse large B cell lymphoma: a SEER- based study. Front Oncol. 2020;10:1093. doi:10.3389/fonc.2020.01093 


\section{Publish your work in this journal}

Cancer Management and Research is an international, peer-reviewed open access journal focusing on cancer research and the optimal use of preventative and integrated treatment interventions to achieve improved outcomes, enhanced survival and quality of life for the cancer patient.
The manuscript management system is completely online and includes a very quick and fair peer-review system, which is all easy to use. Visit http://www.dovepress.com/testimonials.php to read real quotes from published authors. 